Scheduling a Make-To-Stock Queue:

Index Policies and Hedging Points

Michael H. Veatch and Lawrence M. Wein

OR 266-92

September 1992 



\title{
SCHEDULING A MAKE-TO-STOCK QUEUE: INDEX POLICIES AND HEDGING POINTS
}

\author{
Michael H. Veatch \\ Operations Research Center, M.I.T. \\ Lawrence M. Wein \\ Sloan School of Management, M.I.T.
}

\begin{abstract}
A single machine produces several different classes of items in a make-to-stock mode. We consider the problem of scheduling the machine to regulate finished goods inventory, minimizing holding and backorder or holding and lost sales costs. Demands are Poisson, service times are exponentially distributed, and there are no delays or costs associated with switching products. A scheduling policy dictates whether the machine is idle or busy, and specifies the job class to serve in the latter case. Since the optimal solution can only be numerically computed for problems with several products, our goal is to develop effective policies that are computationally tractable for a large number of products. We develop index policies to decide which class to serve, including Whittle's "restless bandit" index, which possesses a certain asymptotic optimality. Several idleness policies, which are characterized by hedging points, are derived, and the best policy is obtained from a heavy traffic diffusion approximation. Nine sample problems are considered in a numerical study, and the average suboptimality of the best policy is less than $3 \%$.
\end{abstract}

September 1992 

In a make-to-stock production facility with multiple products, one of the goals of the scheduling policy is to regulate finished goods inventory. Too small of an inventory risks incurring backorder or lost sales costs, while too large of an inventory increases holding costs. The target inventory level, called the base or safety stock, is vitally linked to randomness in the system and capacity constraints that limit the ability to respond to unexpected demand. Accordingly, a realistic model of the maketo-stock system should include queueing effects. The queueing framework combines the dynamic stochastic nature of the scheduling problem, often studied in inventory systems, with the capacity constraint, usually dealt with through deterministic production scheduling.

Although make-to-order environments, where production occurs after customer orders are received, have been analyzed extensively as queueing control problems (see, for example, Klimov 1974), little work has been done on the problem of scheduling a multiclass make-to-stock queue. Wein (1992) develops a scheduling policy for the make-to-stock system based on a heavy traffic approximation that results in a Brownian motion control problem. Zheng and Zipkin (1990) and Zipkin (1992) propose and analyze a simple "longest queue" policy that is optimal for a system with identical product classes operating under independent base stock policies. Zipkin (1990) proposes yet another policy, attempting a compromise between the very different Brownian and longest queue policies. Ha (1992) partially characterizes the optimal policy for the two-product case. While promising computational results have been reported for the Brownian and longest queue policies, no performance guarantees are available and both have obvious deficiencies in the structure of the control.

This paper develops and tests several new scheduling policies. The system considered is a multiclass $M / M / 1$ make-to-stock queue. Preemptive resume scheduling is allowed and no set-up costs or times are incurred when switching classes. In the backorder version of the problem, which was addressed in the references cited above, the objective is to minimize holding and backorder costs. A lost sales problem is also 
considered, where demands that cannot be met from inventory are lost and a cost incurred. A long-run average cost criterion is used.

Three index policies are considered, where an index is computed for each class as a function of the inventory in that class. The class with the smallest index is served; if all indices are positive, the server is idle. Index policies have the advantage of being computationally tractable even for a large number of classes. An index policy proposed in Zipkin (1990) performed best among those considered. The most innovative, and one that also performs well, is a "restless bandit" index, defined for a general problem in Whittle (1988). This index has the property that it is asymptotically optimal as the number of classes goes to infinity (and the utilization of each class goes to zero). We also discuss the Gittens index for this problem, neglecting its "restlessness," and point out a connection between the Gittens and restless bandit indices. A third index is developed by computing the value function for the system under a naive random policy.

We will show that index policies perform well at determining which class to serve, but do poorly at deciding when to idle. The problem is that each index is computed without knowledge of the other classes, and hence, without knowing the system utilization. Several other approximations are proposed for when to idle. One method decomposes the system into single-class subproblems with the same utilization as the original system. Another aggregates the system into a single product class. The most elegant, and the most accurate, idling decision is derived using a heavy traffic diffusion approximation. We analyze the approximating Brownian control problem for the lost sales case, complementing the backorder case treated in Wein. A fourth idleness policy is derived by computing the inventory distribution assuming that the longest queue policy is used, and then decomposing into single-class subproblems.

Numerical results are presented that compare all of the proposed policies with optimal policies for two- and three-product problems. Combinations of index and 
idleness policies are found that perform well; the average suboptimality for our best policy is $2 \%$ for 6 lost sales problmes and $4 \%$ for 3 backorder problems. The structure of optimal policies is also investigated.

The rest of the paper is organized as follows. Section 1 formulates the problem mathematically and discusses the structure of scheduling policies. Index policies are derived in Section 2 and hedging points (idleness policies) in Section 3. Numerical results are presented in Section 4, and some concluding remarks are made in Section 5.

\section{Dynamic Scheduling Problem}

Consider a multiclass, make-to-stock $\mathrm{M} / \mathrm{M} / 1$ queueing system: a server can produce $K$ different classes of items; each finished item is placed in its respective inventory, $X_{k}(t)$ for class $k, k=1, \ldots, K$ at time $t$; this inventory services an exogenous demand. In the backorder version of the problem, demand that cannot be met from inventory is backordered and recorded as negative inventory. In the lost sales problem, class $k$ demands that occur when $X_{k}(t)=0$ are ignored and a cost incurred. We have several reasons for considering the lost sales problem. It has received less attention than the backorder problem in the literature, is at least as appropriate in many applications, provides an interesting example of Brownian motion analysis, and is the only version to which the restless bandit index of Section 2.3 applies. This index is unique in possessing some form of asymptotic optimality.

The demands for each class are independent Poisson processes with rates $\lambda_{k}$ and the service times for class $k$ items are independent and exponentially distributed with mean $m_{k}=1 / \mu_{k}$. In Section 3.3, we will briefly consider general interdemand and service time distributions. For the backorder problem, stability of the system requires 
that $\rho=\sum \rho_{k}<1$, where $\rho_{k}=\lambda_{k} / \mu_{k}$ (all indices range over $1, \ldots, K$ unless otherwise noted).

The scheduling decision is whether to produce product $1, \ldots, K$ or to idle at each time $t$. An admissible scheduling policy $\pi$ is a function $\zeta(X, t)$ that takes on the values $0,1, \ldots, K$ (zero denoting idle) and is nonanticipating with respect to $X$. Let $\Pi$ denote the class of admissible policies. Production of an item can be interrupted and resumed; no set-up costs or times are incurred when switching from one class to another. Because the system is memoryless, a Markov policy, depending only on the current state $X(t)=\left(X_{1}(t), \ldots, X_{K}(t)\right)$, will be optimal. Consequently, the policy space can be enlarged to include multiple servers or the allocation of partial service effort without changing the problem.

The objective is to minimize holding and backorder costs, incurred at the rate $c^{B O}(x)=\sum\left(h_{k} x_{k}^{+}+b_{k} x_{k}^{-}\right)$in state $x$, for the backorder problem, or holding and lost sales costs, $c^{L S}(x)=\sum\left(h_{k} x_{k}^{+}+s_{k} 1_{\left\{x_{k}=0\right\}}\right)$, for the lost sales problem. Note that $s_{k}$ is the cost rate for a stockout. The expected cost per lost sale is $s_{k} / \lambda_{k}$. The infinitehorizon cost, discounted at the rate $\alpha>0$, is $V^{\pi}(x)=E_{x} \int_{0}^{\infty} e^{-\alpha t} c(X(t)) d t$. Here $E_{x}$ denotes expectation given the initial state $X(0)=x$ and policy $\pi$. Let $\bar{\mu}=\max _{k}\left\{\mu_{k}\right\}$ and $\Lambda=\sum \lambda_{k}+\bar{\mu}+\alpha$. The optimal cost function, $V(x)=\min _{\pi \in \Pi} V^{\pi}(x)$, satisfies the dynamic programming optimality equations

$$
\begin{gathered}
V(x)=\mathbf{T} V(x), \\
\mathbf{T} V(x)=\frac{1}{\Lambda}\left[c(x)+\sum \lambda_{k} V\left(x-e_{k}\right)\right. \\
\left.+\min \left\{\bar{\mu} V(x), \min _{k}\left\{\mu_{k} V\left(x+e_{k}\right)+\left(\bar{\mu}-\mu_{k}\right) V(x)\right\}\right\}\right],
\end{gathered}
$$

where $e_{k}$ is the unit vector with $k$ th component equal to one. For the lost sales problem, replace $x-e_{k}$ with $x$ in (2) when $x_{k}=0$. It is more convenient to deal with an undiscounted, long-run average cost criterion. In this case $\alpha=0$; the optimal 
average cost rate (gain) $g$ and relative value function $V(x)$ satisfy

$$
V(x)+g=\mathbf{T} V(x)
$$

where we arbitrarily set $V(0)=0$.

The following characterization of Markov policies will be useful. Let $\mathcal{B}_{k}$ be the set of states in which class $k$ is produced and $\mathcal{I}$ the set in which the server is idle. We consider only policies having the monotonicity property that $x+a e_{k} \in \mathcal{B}_{k}$ if and only if $a<A$, for some $-\infty \leq A \leq \infty$. For such policies, each pair $\mathcal{B}_{j}, \mathcal{B}_{k}$ can be separated by a switching surface that is nondecreasing (when viewed as a function of any $K-1$ of the state variables $x_{k}$ ). Roughly speaking, the intersection of all of the switching surfaces forms a switching curve. To be more precise, define the switching curve as a sequence of points $\left\{x_{n}, n=1, \ldots, N\right\} \in \mathbf{Z}^{k}$ such that

1. $x^{n+1}=x^{n}+e_{k}$, where $x^{n} \in \mathcal{B}_{k}$,

2. $x^{N} \in \mathcal{I}$, and

3. for every $x^{n} \in \mathcal{B}_{k}$ and $j \neq k$, either $x^{n}-e_{j} \in \mathcal{B}_{j}$ or, for the lost sales problem, $x_{j}^{n}=0$.

The final point in the switching curve is called the hedging point, denoted $x^{*}=x^{N}$; its components $x_{k}^{*}$ are the target or base stock levels. Starting from any point not in $\mathcal{I}$, the system without arrivals moves toward the switching curve, then along it to the hedging point, and then stops.

On its recurrent states, a policy can be defined by the switching surfaces and hedging point. To see the role of each more clearly, eliminate the idleness option and augment $\mathcal{B}_{k}$ to include the (previously idle) states in which class $k$ is served, defining new switching surfaces and an unbounded switching curve $(N=\infty)$. These surfaces 
define the preference among classes. The hedging point (on the switching curve) defines the idleness region. The combination of switching surfaces (just a switching curve for two classes) and hedging point fully specifies a policy.

Optimal policies for this problem can only be found numerically, and only when the number of classes is small. Hence, we are led to consider approximate policies. Several classes of policies have been considered in other papers. One possible set of switching surfaces is the non-Markov policy considered by Zheng and Zipkin that services demands FCFS given a hedging point. They also consider a longest queue (LQ) policy that serves the class with the most demands in the demand queue. Zheng and Zipkin consider two identical products, so that the LQ policy (which is symmetrical for $x_{1}^{*}=x_{2}^{*}$ ) is optimal, and find that LQ is only marginally better than FCFS. For two non-identical products, we call a switching curve that lies along $x_{1}=x_{2}$ for $x_{k} \leq \min \left\{x_{1}^{*}, x_{2}^{*}\right\}$, then extends vertically or horizontally to the hedging point, an LQ switching curve. An offset LQ switching curve lies along $x_{1}=x_{2}+\left(x_{1}^{*}-x_{2}^{*}\right)$. However, the offset $L Q$ switching curves tested in Section 4.2 are modified, if necessary, for consistency with the following property. Ha (1992) proves that, in the two-product case, it is optimal to serve the class $k$ with maximal $b_{k} \mu_{k}$ when it is backordered; i.e., the switching curve cannot have $x_{k}<0$ for this class.

Wein proposes a policy for the backorder problem that is suggested by a heavy traffic analysis and reminiscent of the $c \mu$ rule: if there are classes in danger of being backordered (i.e., $X_{k}(t)<\epsilon_{k}$, where $\epsilon_{k}$ is discretionary), serve the class within this set with the largest $b_{k} \mu_{k}$; otherwise, serve the class with the smallest $h_{k} \mu_{k}$ for which $X_{k}(t)<x_{k}^{*}$. The hedging point $x^{*}$ is obtained as the solution to a Brownian control problem.

The primary goal of this paper is to develop more sophisticated, yet easily computable, switching curves/surfaces and additional hedging point approximations that perform better than the policies described above. 


\section{Index Policies}

The class of index policies is attractive because its computational complexity only grows linearly in the number of classes. An index policy is defined by assigning an index $\nu_{k}\left(x_{k}\right)$ to class $k$ and serving the class with smallest index. If the indices are nondecreasing, the policy has the monotonicity property of Section 1 and has a switching curve. A hedging point must also be defined. We will call a policy pure index if the idleness region is $\mathcal{I}=\left\{x: \nu_{k}\left(x_{k}\right) \geq 0\right.$ for all $\left.k\right\}$; the hedging point is the first point on the switching curve that is in $\mathcal{I}$. Otherwise, the hedging point will be specified in addition to the index.

Since the minimum in (2) is achieved by a class $k$ for which $\mu_{k} \Delta_{k} V(x)$ is minimal, where $\Delta_{k} V(x)=V\left(x+e_{k}\right)-V(x)$, the goal of any index policy is to rank-order $\left\{\mu_{k} \Delta_{k} V(x)\right\}$. Several indices, listed in Table 1, are developed and tested in this paper. The most obvious approach is to approximate the optimal value function $V(x)$. In Section 2.1, $\Delta_{k} V(x)$ is approximated using the value function for separate single-product problems where service capacity is allocated across product classes. In the lost sales problem, a hedging point must be specified before this index can be computed. The service time look-ahead (STLA) index of Section 2.2, proposed by Zipkin (1990), replaces $V(x)$ with the expected cost rate after one service time. It is related to the fully myopic policy of using the cost rate after one transition, which produces the $b \mu$ and $h \mu$ rule. The last index is based on a Lagrangian approach to the multi-armed "restless" bandit problem in Whittle.

\subsection{Value Function Approximation}

In this section, we derive an index for backorders and lost sales, using a value function approximation. For brevity, this index is called $\mu \Delta V$. Consider a single-product 
Table 1: Proposed Indices and Hedging Points.

$\underline{\text { Index }}$

Backorders Lost Sales

Value function approx. $(\mu \Delta V)$

Service time look-ahead (STLA)

Restless bandit

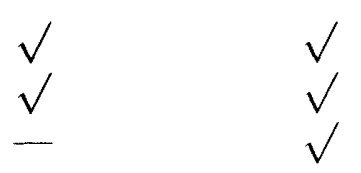

\section{Hedging Point}

Server allocation

Aggregate product

hedging point hedging point

workload workload

Longest queue (LQ) hedging point hedging point

Brownian motion

workload

workload

subproblem for class $k$ with the service rate $\left(\rho_{k} / \rho\right) \mu_{k}$. In this problem, the server's availability in any time interval has been allocated across classes in proportion to their utilization $\rho_{k}$. One motivation for this allocation is that it changes the subproblem utilization from $\rho_{k}$, which tends to zero as the number of classes increases, to the system utilization $\rho$. Another motivation is that this fixed allocation can be viewed as a crude static policy. Selecting the minimal $\mu \Delta V$ under this policy is equivalent to performing one value iteration, giving an improved policy.

We solve the allocated server subproblem for the undiscounted backorder case first. Drop the subscript $k$, let $\mu$ be the allocated service rate, and let $V(x)$ and $g$ be the optimal relative value function and gain for this problem, satisfying

$$
\begin{gathered}
V(x)+g= \\
\frac{1}{\Lambda} \min \{c(x)+\lambda V(x-1)+\mu V(x+1), c(x)+\lambda V(x-1)+\mu V(x)\} .
\end{gathered}
$$

The optimal policy will be of threshold form $\pi(B)$ for some $B>0$ : the server is busy in states $x<B$ and idle in states $x \geq B$. Under $\pi(B), B-X$ is an $M / M / 1$ queue. Letting $f(\cdot)$ and $F(\cdot)$ be the steady-state p.d.f. and d.f. of $B-X$, the corresponding 
gain is

$$
\begin{aligned}
\Lambda g^{\pi(B)} & =\sum_{x=-\infty}^{B} c(x) \operatorname{Pr}\{X=x\} \\
& =\sum_{x=0}^{B-1} h(B-x) f(x)+\sum_{x=B+1}^{\infty} b(x-B) f(x)
\end{aligned}
$$

Differencing,

$$
\begin{aligned}
\Lambda\left(g^{\pi(B+1)}-g^{\pi(B)}\right) & =\sum_{x=0}^{B} h f(x)+\sum_{x=B+1}^{\infty} b f(x) \\
& =(h+b) F(B)-b .
\end{aligned}
$$

The d.f. $F$ is nondecreasing, so $g^{\pi(B)}$ is convex. It attains its minimum at the smallest $B$ for which (6) is positive, namely $B=\min \{x: F(x)>b /(h+b)\}$. Since $F(x)=1-\rho^{x+1}$, it folows that

$$
B=\lceil\ln [h /(h+b)] / \ln \rho\rceil-1
$$

We will also need the optimal gain. Evaluating (6) using $f(x)=(1-\rho) \rho^{x}$ gives

$$
\Lambda g=\frac{h B-h(B+1) \rho+(h+b) \rho^{B+1}}{1-\rho} .
$$

The dynamic programming equations are

$$
\begin{gathered}
V(x)+g=\frac{1}{\Lambda}[c(x)+\lambda V(x-1)+\mu V(x+1)], \quad x<B, \text { and } \\
V(x)+g=\frac{1}{\Lambda}[c(x)+\lambda V(x-1)+\mu V(x)], \quad x \geq B .
\end{gathered}
$$

We can recursively compute $\Delta V(x)$ from the equations

$$
\Delta V(x)=[h(x+1)-\Lambda g] / \lambda, \quad x \geq B-1, \text { and }
$$




$$
\Delta V(x)=\frac{c(x+1)-\Lambda g}{\lambda}+\frac{\Delta V(x+1)}{\rho}, x<B-1 .
$$

The index is $\nu(x)=\mu \Delta V(x)$.

The same approach is used for the lost sales problem, except that $B$ must be specified separately, i.e., a hedging point is needed. The recursion obtained is

$$
\begin{aligned}
\Delta V(0) & =(\Lambda g-s) / \mu \text { and } \\
\Delta V(x) & =\frac{\Lambda g-h x}{\mu}+\rho \Delta V(x+1), 0<x<B, \text { where } \\
g & =\left\{s \rho^{B} P_{B}+h P_{B}\left[\frac{B-(B+1) \rho+\rho^{B+1}}{(1-\rho)^{2}}\right]\right\} / \Lambda \text { and } \\
P_{B} & =\frac{1-\rho}{1-\rho^{B+1}} .
\end{aligned}
$$

Solving the recursion gives

$$
\mu \Delta V(x)=-\frac{s \rho^{x}\left(1-\rho^{B-x}\right)}{1-\rho^{B+1}}+\frac{h\left[B-x-(B+1) \rho^{x+1}+(x+1) \rho^{B+1}\right]}{\left(1-\rho^{B+1}\right)(1-\rho)} .
$$

\subsection{Service Time Look-Ahead}

Static priority policies such as the $h \mu$ rule are fully myopic in the sense that they minimize the cost rate $c(\cdot)$ of the next state. Zipkin (1990) proposes a service time look-ahead (STLA) policy that considers the expected cost rate after one service time. It can be viewed as a myopic policy with no preemption. No preemption forces some degree of looking ahead, and so is preferable to only considering the cost rate of the next state. This policy is reminiscent of the transportation time look-ahead policy of Miller (1974) for the decision of which base to send an item repaired at a central depot, where transportation time takes on the role of service time.

For a given product, let $g(x)=E[c(x-D(S))]$, where $S$ is the service time and 
$D(t)$ is the number of demands in the interval $(0, t]$. Then $g(x)$ is the expected cost rate after one service time if the server is idle. If the server is busy, the cost is $g(x+1)$; hence, the rate at which serving this class increases the expected cost rate, assuming no preemption, is $\nu(x)=\mu \Delta g(x)$. Zipkin's policy is to choose the class with smallest $\nu(x)$, so it is an index policy. We also consider the pure index policy of idling when $\nu(x)>0$ for all classes.

Zipkin evaluates the index for the backorder problem using the fact that $D(S)+1$ has a geometric distribution with parameter $p=\mu /(\lambda+\mu)$. Letting $q=1-p$, $\operatorname{Pr}\{D(S)=j\}=q^{j} p, j \geq 0$ and for $x \geq 0$

$$
\begin{aligned}
g(x) & =h \sum_{j=1}^{x} j q^{x-j} p+b \sum_{x=1}^{\infty} j q^{x+j} p \\
& =h\left(x p-q+q^{x+1}\right) / p+b q^{x+1} / p .
\end{aligned}
$$

The index is

$$
\mu \Delta g(x)=-b \mu q^{x+1}+h \mu\left(1-q^{x+1}\right), \quad x \geq 0 .
$$

Note that, as $x \rightarrow \infty,(19)$ approaches $h \mu$, and for large inventories the STLA policy gives the $h \mu$ rule. For $x<0, g(x)=E[b(-x+D(S))]=b(-x+q / p)$, and the index is

$$
\mu \Delta g(x)=-b \mu, \quad x<0,
$$

which yields the $b \mu$ rule. Turning to the lost sales problem,

$$
\begin{aligned}
g(x) & =s \sum_{j=x}^{\infty} q^{j} p+h \sum_{j=1}^{x} j q^{x-j} p \\
& =s q^{x}+h\left(\frac{x p-q+q^{x+1}}{p}\right) .
\end{aligned}
$$

The index is

$$
\mu \Delta g(x)=-s \mu p q^{x}+h \mu\left(1-q^{x+1}\right) .
$$




\subsection{Restless Bandit Analysis}

In this section, we use Whittle's work on "restless bandits" to obtain an index for the lost sales problem and analyze its properties. Whittle defines a restless bandit problem as a resource allocation problem similar to a multi-armed bandit except that the arms not being played, called passive, continue to change state according to a Markov law that is different than the law governing their transitions when active. Passive arms can also incur costs. In the scheduling problem, there are $K+1$ arms, one for each class plus an idleness arm whose index is zero. There must be exactly one active arm at each time, where active means that the server is assigned to that class. An index is defined by considering a single-product subproblem that chooses between active (serving) or passive (idling) given a passive tax, or cost of not serving, $\nu$. We will consider only the undiscounted, lost sales problem. Let $\Lambda_{k}=\lambda_{k}+\mu_{k}$. Dropping the subscript $k$, the optimal cost function $V(x, \nu)$ and gain $g(\nu)$ satisfy the optimality equations

$$
\begin{gathered}
V(x, \nu)+g(\nu)= \\
\frac{1}{\Lambda} \min \{c(x)+\lambda V(x-1, \nu)+\mu V(x+1, \nu), \nu+c(x)+\lambda V(x-1, \nu)+\mu V(x, \nu)\} .
\end{gathered}
$$

When $x=0$, replace $x-1$ with $x$ in (23). The optimal policy will be of threshold form, active in states $x=0, \ldots, B-1$ and passive when $x \geq B$. Denote this policy $\pi(B)$, with the corresponding gain $g^{\pi(B)}$. Under $\pi(B), B-X$ is a finite queue with utilization $\rho$ (all notation now refers to a single class). Assuming that $\rho<1$, its steady-state distribution is $P_{B}(x)=\operatorname{Pr}\{X=x \mid \pi(B)\}=\rho^{B-x} P_{B}$, where $P_{B}=P_{B}(B)=(1-\rho) /\left(1-\rho^{B+1}\right)$. The restless bandit index $\nu(x)$ is defined as the passive tax that achieves indifference in the min in (23), i.e., $\Delta V(x, \nu(x))=\nu(x) / \mu$, where $\Delta V(x, \nu)=V(x+1, \nu)-V(x, \nu)$. Indifference in (23) at $x=B-1$ implies that the policies $\pi(B-1)$ and $\pi(B)$ are both optimal. Hence, the index $\nu(B-1)$ can be found by solving for $\nu$ in

$$
g^{\pi(B-1)}(\nu)=g^{\pi(B)}(\nu)
$$


For a given policy, the contribution of $\nu$ to the gain (per transition) is

$$
g^{\pi(B)}(\nu)=g^{\pi(B)}(0)+P_{B} \nu / \Lambda
$$

Combining (24) and (25),

$$
\nu(B-1)=\frac{\Lambda\left[g^{\pi(B)}(0)-g^{\pi(B-1)}(0)\right]}{P_{B-1}-P_{B}} .
$$

The gain is

$$
\begin{aligned}
\Lambda g^{\pi(B)}(0) & =\sum_{x=0}^{B} P_{B}(x) c(x) \\
& =s P_{B}(0)+h \sum_{x=1}^{B} x P_{B}(x) \\
& =s \rho^{B} P_{B}+h P_{B} \rho^{B} \sum_{x=1}^{B} x(1 / \rho)^{x} \\
& =s \rho^{B} P_{B}+h P_{B}\left[\frac{B-(B+1) \rho+\rho^{B+1}}{(1-\rho)^{2}}\right] .
\end{aligned}
$$

The denominator of (26) can be written $\rho^{B} P_{B-1} P_{B}$, and after further simplification, (26) becomes

$$
\nu(B-1)=-\frac{s}{\rho}+\frac{h\left[1-(B+1) \rho^{B}+B \rho^{B+1}\right]}{(1-\rho)^{2} \rho^{B}} .
$$

Notice that the lost sales term in (28) is constant, meaning that the penalty paid for lost sales in this index scheme is the same whether lost sales are being incurred $(x=0)$ or not $(x>0)$. We can understand why the $s$ term is constant by viewing $x=0$ as a renewal point with a certain reward. Discounting would change this result. The lost sales term tends to dominate the index for small inventory positions (less than the hedging point), and the class with minimal $s_{k} \mu_{k} / \lambda_{k}$ is produced when $x$ is small. This min $s \mu / \lambda$ rule will be seen again in Section 3.3. For large inventory positions (beyond the hedging point), the switching curve is approximately a straight line with slope $\mathrm{d} x_{i} / \mathrm{d} x_{j}=\ln \rho_{i} / \ln \rho_{j}$, which is a weighted version of the LQ policy. 
Unlike the Gittens index for the standard multi-armed bandit problem, the restless bandit index does not give an optimal policy. Under certain conditions, however, an asymptotic optimality holds. Let $\mathbf{X}$ be the state space for an arm and $D(\nu) \subseteq \mathbf{X}$ be the set of states in which the optimal policy for $\operatorname{tax} \nu$ is active.

Definition 1. An arm is indexable if $D(\nu)$ increases monotonically from $\emptyset$ to $\mathbf{X}$ as $\nu$ increases from $-\infty$ to $\infty$.

Consider a problem with the constraint that exactly $m$ of $n$ arms must be active at any one time, and a relaxed-constraint problem where a time average of $m$ arms must be active. If all arms are indexable, then as $m, n \rightarrow \infty$ with $m / n$ fixed, the optimal per-project gain is asymptotically the same as that for the relaxed-constraint problem. Furthermore, Whittle conjectures and Weber and Weiss (1990) prove under an additional technical condition that the index policy defined above is asymptotically optimal for the relaxed-constraint problem. Hence, the index policy is asymptotically optimal for the exactly- $m$ problem.

To establish indexability for the lost sales problem, one needs to show that $\nu(x)$ is nondecreasing and well-defined for all $x$. These properties follow from (28). However, the above result is for problems with $m \rightarrow \infty$ active arms, i.e., $m$ servers. It says that, for an $m$-server $K$-class problem (with $K+m$ arms), the index policy is approximately optimal for a large number of servers and classes. The one-server problem differs from an $m$-server problem with service rates divided by $m$ only in the higher service rate that can be applied to a class. If $K \rho_{k}$ is bounded for all $k$ as $K \rightarrow \infty$, i.e., each product has a small utilization, then the service rate limit should be irrelevant and it is reasonable to expect that asymptotic optimality holds for the one-server problem with a large number of classes.

In contrast, the backorder problem is not indexable; $\nu(x)$ does not exist (i.e., equals $-\infty$ ) for all $x$. The difficulty is that $\nu$ is a Lagrange multiplier for the constraint on the time-average number of active arms. For the backorder problem, any stable 
policy must serve a time-average of $\rho$ classes, so relaxing this constraint does not change the optimal value and the Lagrange multiplier does not exist.

A second characteristic of the restless bandit index is that the hedging point $x^{*}$ defined by the pure index policy lies on the boundary of the optimal idleness region, $x_{k}^{*}=\min \left\{x_{k}: x \in \mathcal{I}\right\}$, so that $x^{*}$ is a lower bound on the optimal hedging point. Monotonicity arguments imply that $\mathcal{I}$ touches the "asymptotes" $x_{k}=x_{k}^{*}$ for large $x$.

\section{Hedging Points}

Several hedging point approximations, listed in Table 1, are also developed and tested. Recall that one can use either a pure index policy or an index policy plus a hedging point approximation. The server allocation method allocates service capacity across classes and then solves each single-product problem to find its base stock level $x_{k}^{*}$. A second method is to aggregate into a single product. Instead of stock levels, a threshold is found on the total workload, or expected service time, represented by the stock. Given a switching curve, the workload threshold defines a hedging point. Both of these methods reduce to solving a single-product problem, which is done in Section 3.1. A third method (Section 3.2) uses the analysis of the LQ policy for the backorder case in Zipkin (1992). For each product, the variance of the demand queue under an LQ policy is approximated, a distribution fit to the variance, and a stock level computed. A workload threshold can also be found through a heavy traffic diffusion approximation. The backorder problem is analyzed in Wein; we treat the lost sales problem in Section 3.3. Finally, if the performance of a policy can be evaluated, the best hedging point for a given index policy can be found by searching along the switching curve. 


\subsection{Single-Product Methods for Hedging Points}

In this section we develop two hedging point approximations that construct and solve single-product subproblems. The first method, called allocated server, creates a subproblem for each class by allocating the server as in Section 2.1. The subproblem parameters for class $k$ are $\mu_{k}^{\text {all }}=\left(\rho_{k} / \rho\right) \mu_{k}, \rho_{k}^{\text {all }}=\rho$, and $\lambda_{k}, h_{k}, b_{k}$, and $s_{k}$ unchanged. The second method, called aggregate product, aggregates all products into a single class. The subproblem parameters are $\lambda^{\mathrm{agg}}=\sum \lambda_{k}, \mu^{\mathrm{agg}}=\sum \lambda_{k} / \rho, \rho^{\mathrm{agg}}=\rho, h^{\mathrm{agg}}=$ $\sum\left(\rho_{k} / \rho\right) h_{k}, b^{\text {agg }}=\sum\left(\rho_{k} / \rho\right) b_{k}$, and $s^{\text {agg }}=\sum\left(\rho_{k} / \rho\right) s_{k}$. To interpret stock levels for this aggregate product, we use the workload concept of Section 3.3. A stock level of $B$ represents an expected service time, or workload, of $w=B / \mu^{\text {agg }}$. This workload can then be combined with a switching curve to set stock levels for the original products.

Before solving these subproblems, we comment on the nature of the approximations. Decomposing the system into single-product problems with the same utilization is analogous to decomposing a multi-server queue into parallel single-server queues. Performance degrades, total queue length increases, and the optimal stock level increases to compensate. Thus, the allocated server hedging point should be larger than optimal. Aggregating the system into a single product neglects the variation in $X_{k}$ given $\sum X_{k}$, i.e., the inability to maintain the desired allocation of inventory among classes. Because variability is neglected, this hedging point should be smaller than optimal.

We will solve a generic subproblem, dropping the superscripts and subscripts from the parameters. The backorder case has already been solved; the optimal stock level is given by (7). For the lost sales case, let $\Delta g(B)=g^{\pi(B+1)}-g^{\pi(B)}$, where $g^{\pi(B)}$ is the gain under policy $\pi(B)$. From (15),

$$
\Lambda \Delta g(B)=s \rho^{B}\left(\rho P_{B+1}-P_{B}\right)
$$




$$
+h\left\{\frac{P_{B+1}\left[B+1-(B+2) \rho+\rho^{B+2}\right]-P_{B}\left[B-(B+1) \rho+\rho^{B+1}\right]}{(1-\rho)^{2}}\right\},
$$

where $P_{B}$ is given by (16). We wish to find the stock level $B$ that minimizes $g^{\pi(B)}$. It can be shown that $g^{\pi(B)}$ is concave; hence, $B=\min \{x: \Delta g(x) \geq 0\}$. A search over the values $x=0,1, \ldots$ is used to find $B$.

\subsection{Hedging Points Based on Longest Queue Policies}

Zipkin (1992) analyzes the longest queue (LQ) policy for identical products in the undiscounted, backorder problem. In this section, we use his result to approximate the case of non-identical products. The corresponding policy is offset LQ. The steadystate distribution of $X_{k}$ is then used to find the hedging point. This LQ hedging point involves five approximations:

1. Assume an offset LQ policy is optimal,

2. Use Zipkin's approximation of the inventory variance for identical products,

3. Adjust for non-identical products,

4. Fit a distribution to the mean and variance, and

5. Decompose the idleness decision into single-product subproblems.

Step (2) is exact for two products and (1) is exact for identical products. We also tested the accuracy of steps (3) and (4) for two products by computing the joint p.d.f. of the inventories as outlined in Section 8 of Zheng and Zipkin. The j.p.d.f. method gave slightly larger hedging points but, due to compensating approximation errors, resulted in worse values of the gain $g$. The j.p.d.f. method is not described here or used in Section 4. 
Zipkin's variance estimate is a function of the number of identical products. For products with $\lambda_{j} \neq \lambda_{k}$ or $\mu_{j} \neq \mu_{k}$, the fraction of utilization due to class $k$ is $\alpha_{k}=\rho_{k} / \rho$. Define the effective number of products for class $k$ as $K_{k}^{\text {eff }}=1 / \alpha_{k}$, i.e., the number of identical products needed to achieve the system utilization. Notice that for identical products $K_{k}^{\text {eff }}=K$. The steady-state variance of $X_{k}$ is

$$
\sigma_{k}^{2}=\frac{\operatorname{Var}(N)+E\left(D_{k}^{2}\right)}{\left(K_{k}^{\mathrm{eff}}\right)^{2}}
$$

where $N$ is the aggregate demand queue for $K_{k}^{\text {eff }}$ identical products, each with demand queue $N_{k}(t)=x_{k}^{*}-X_{k}(t)$, and $E\left(D_{k}^{2}\right)$ captures variation in $N_{k}$ given $N$. Since $N$ is the number of customers in an $M / M / 1$ queue, $\operatorname{Var}(N)=\rho /(1-\rho)^{2}$. Zipkin uses the approximation

$$
E\left(D_{k}^{2}\right)=\left(K_{k}^{\mathrm{eff}}-1\right) \rho\left[1+\rho+\left(1-2 \alpha_{k}\right) \rho^{2}+\left(1-2 \alpha_{k}\right)^{2} \rho^{3}\right] .
$$

Also, estimate the mean of $N_{k}$ as

$$
E\left(N_{k}\right)=\frac{E(N)}{K_{k}^{\text {eff }}}=\frac{\alpha_{k} \rho}{1-\rho}=\frac{\rho_{k}}{1-\rho},
$$

which is exact for identical products.

Given the first two moments of $N_{k}$, we will fit a distribution to them in order to calculate the upper percentiles on which the stock level depends. Since $N$ is geometric, it is reasonable to use a distribution that has a geometric tail. For simplicity, we use the shifted geometric distribution $f(x)=p q^{(x-a)-1}, x=a+1, a+2, \ldots$. Fitting to (30) and (32) gives

$$
\begin{gathered}
q=1-\frac{\sqrt{4 \sigma_{k}^{2}+1}-1}{2 \sigma_{k}^{2}} \text { and } \\
a=E\left(N_{k}\right)-\frac{1}{1-q} .
\end{gathered}
$$


The optimal stock level has already been found for a geometric distribution; (7) gives

$$
B=\lceil\ln [h /(b+h)] / \ln q\rceil-1+a .
$$

\subsection{Brownian Motion Analysis}

Wein develops a policy for the backorder problem using a diffusion approximation. The server is idle when the workload $W(t)=\sum m_{k} X_{k}(t)$ exceeds the threshold

$$
c=\frac{\sum \lambda_{k} m_{k}^{2}\left(v_{s k}^{2}+v_{d k}^{2}\right)}{2(1-\rho)} \ln (1+b / h),
$$

where $b=\min \left\{b_{k} \mu_{k}\right\}, h=\min \left\{h_{k} \mu_{k}\right\}$, and $v_{s k}$ and $v_{d k}$ are the service and interdemand time coefficients of variation for class $k$. In the case of exponential distributions, $v_{s k}=v_{d k}=1$. The switching curve is determined by the $b \mu$ and $h \mu$ rule, so that all of the workload is held in the class with minimal $h_{k} \mu_{k}$ (actually, a small discretionary amount can be held in every other class).

We derive an analogous policy for the lost sales problem. The essential difference is that the heavy traffic condition, $\rho$ slightly less than one, is replaced by the conditions $\rho \approx 1$ and the ratio of holding to lost sales cost is small, $h / s \ll 1$. The approach taken is to (1) formulate the scheduling problem in terms of cumulative processes, (2) define an approximate Brownian motion control problem, (3) reformulate to give a more tractable control problem called the workload formulation, (4) solve the workload formulation for an initial throughput, and (5) calculate a new throughput from this solution and iterate until the throughput converges. For consistency with much of the heavy traffic scheduling literature, inventory will be denoted $Z_{k}(t)$, not $X_{k}(t)$, in this section. 


\subsubsection{The Scheduling Problem}

Define the renewal counting processes

$$
\begin{aligned}
S_{k}(t)= & \text { number of class } k \text { service completions after serving class } k \text { for time } t \\
D_{k}(t)= & \text { number of class } k \text { demands that occur during the first } t \text { units of non- } \\
& \text { stockout time, i.e., times } s \text { when } Z_{k}(s)>0
\end{aligned}
$$

with rates and coefficients of variation $\mu_{k}, \lambda_{k}, v_{s k}$, and $v_{d k}$, respectively. It is implicit in this definition of $D_{k}$ that the demand process is turned off during stockouts, i.e., interdemand time is measured with respect to nonstockout time. For exponential distributions, the Poisson demand process of Section 1, which is not turned off, is equivalent. The scheduling problem of Section 1 can be posed as finding processes $T_{k}$ that are nonanticipating with respect to $Z$ to

$$
\min \limsup _{T \rightarrow \infty} \frac{1}{T} E\left[\int_{0}^{T} \sum h_{k} Z_{k}(t) \mathrm{d} t+\sum s_{k} \bar{A}_{k}(T)\right]
$$

subject to

$$
\begin{gathered}
Z_{k}(t)=S_{k}\left(T_{k}(t)\right)-D_{k}\left(A_{k}(t)\right), \\
A_{k}(t)=\int_{0}^{t} 1_{\left\{Z_{k}(s)>0\right\}} \mathrm{d} s, \\
\bar{A}_{k}(t)=t-A_{k}(t), \\
I(t)=t-\sum T_{k}(t), \text { and }
\end{gathered}
$$

$T_{k}$ and $I$ nondecreasing with $T_{k}(0)=0$.

Here $T_{k}(t)$ is the cumulative time that class $k$ is served in $(0, t], I(t)$ is the idle time in $(0, t]$, and $A_{k}(t)$ is the time in $(0, t]$ during which class $k$ demands can arrive. 


\subsubsection{The Brownian Control Problem}

Let $\alpha_{k}=\rho_{k} / \rho$. Following Wein and Dai and Harrison (1991), define $Y_{k}(t)=\alpha_{k} t-$ $T_{k}(t)$ and

$$
X_{k}(t)=S_{k}\left(T_{k}(t)\right)-\mu_{k} T_{k}(t)-\left[D_{k}\left(A_{k}(t)\right)-\lambda_{k} A_{k}(t)\right]+\left(\mu_{k} \alpha_{k}-\lambda_{k}\right) t .
$$

By (38), (40), and (43),

$$
Z_{k}(t)=X_{k}(t)-\mu_{k} Y_{k}(t)+\lambda_{k} \bar{A}_{k}(t)
$$

Note that $\bar{A}_{k}$ increases only when $Z_{k}=0$. Letting $L_{k}(t)=\lambda_{k} \bar{A}_{k}(t)$, we see that $L_{k}$ is the lower regulator for $Z_{k}$, and the term $s_{k} \bar{A}_{k}(T)$ in (37) equals $l_{k} L_{k}(T)$, where $l_{k}=s_{k} / \lambda_{k}$ is the cost per lost sale.

A heavy traffic limit argument can be used to approximate $X_{k}$; see Veatch (1992) for details. We assume the heavy traffic condition that there exists a large integer $n$ such that $\sqrt{n}|1-\rho|$ is small. Notice that, in contrast to traditional open queueing systems, our lost sales case allows for the possibility that $\rho>1$. However, with lost sales, this heavy traffic condition is not sufficient to obtain a limiting Brownian control problem. As in Krichagina, Lou and Taksar (1992), we must also have that the relative cost of lost sales, $l_{k} / h_{k}$, tends to infinity; in particular, their limiting argument assumes that $l_{k} / h_{k}=O(n)$. It is the scaled processes $X_{k}(n t) / \sqrt{n}$, where $n$ is large, for which a limit argument is constructed. Accordingly, we fix $n$ and introduce the scaled processes and cost parameters

$$
\begin{gathered}
\hat{Z}_{k}(t)=\frac{Z_{k}(n t)}{\sqrt{n}}, \quad \hat{X}_{k}(t)=\frac{X_{k}(n t)}{\sqrt{n}}, \quad \hat{Y}_{k}(t)=\frac{Y_{k}(n t)}{\sqrt{n}}, \quad \hat{L}_{k}(t)=\frac{L_{k}(n t)}{\sqrt{n}}, \\
\hat{I}(t)=\frac{I(n t)}{\sqrt{n}}, \quad \hat{h}_{k}=\sqrt{n} h_{k} \quad \text { and } \quad \hat{l}_{k}=l_{k} / \sqrt{n} .
\end{gathered}
$$


For a given policy, define $\gamma_{k}=\lim _{t \rightarrow \infty} T_{k}(t) / t$ and $P_{k}=\lim _{t \rightarrow \infty} \bar{A}_{k}(t) / t$. Then $\gamma_{k}$ is the actual utilization for class $k$, reduced from $\rho_{k}$ by lost sales, and $\mu_{k} \gamma_{k}$ is the class $k$ throughput. The lost sales rate for class $k$ is $\lambda_{k} P_{k}, P_{k}$ is the probability of a stockout, and

$$
\gamma_{k}=\left(1-P_{k}\right) \rho_{k} \leq \rho_{k}
$$

Upon replacing $T_{k}(t)$ and $A_{k}(t)$ in (43) by their mean values $\gamma_{k} t$ and $\left(1-P_{k}\right) t$, as proposed in Harrison (1988), it can be shown by weak convergence arguments that $X_{k}(n t) / \sqrt{n}$ is well approximated by a Brownian motion process. For simplicity of notation, we also use $\hat{X}_{k}$ to denote the Brownian motion. By (43), the drift of the Brownian motion $\hat{X}_{k}$ is $\sqrt{n}\left(\mu_{k} \alpha_{k}-\lambda_{k}\right)$ and the variance of $\hat{X}_{k}$ depends on the policy and equals $\mu_{k} \gamma_{k} v_{s k}^{2}+\lambda_{k}\left(1-P_{k}\right) v_{d k}^{2}=\mu_{k} \gamma_{k}\left(v_{s k}^{2}+v_{d k}^{2}\right)$. In (37), $Z_{k}(t)$ can be replaced by $Z_{k}(n t)$ while $L_{k}(t)$ can be replaced by $L_{k}(n t) / n$, giving

$$
\min \limsup _{T \rightarrow \infty} \frac{1}{T} E\left[\int_{0}^{T} \sum \hat{h}_{k} \hat{Z}_{k}(t) \mathrm{d} t+\sum \hat{l}_{k} \hat{L}_{k}(T)\right] .
$$

The requirement that $T_{k}$ be nondecreasing can be dropped because, for any policy with throughput $\mu_{k} \gamma_{k}>0, T_{k}(n t) / \sqrt{n}$ increases arbitrarily quickly in $t$ for large $n$. Hence, for small $|1-\rho|$ and large $l_{k} / h_{k}$, we are led to approximate the scheduling problem (37)-(43) by the following Brownian control problem (BCP): find processes $\hat{Y}_{k}$ that are nonanticipating with respect to $\hat{X}$ to achieve the objective (48) subject to

$$
\begin{gathered}
\hat{Z}_{k}(t)=\hat{X}_{k}(t)-\mu_{k} \hat{Y}_{k}(t)+\hat{L}_{k}(t), \\
\hat{I}(t)=\sum \hat{Y}_{k}(t), \\
\hat{L}_{k}(t)=-\inf _{0 \leq s \leq t}\left\{\hat{X}_{k}(s)-\mu_{k} \hat{Y}_{k}(s)\right\}, \\
\hat{I} \text { nondecreasing and } \hat{Y}_{k}(0)=0 .
\end{gathered}
$$

The symbols $\hat{Z}_{k}, \hat{Y}_{k}, \hat{L}_{k}$ and $\hat{I}$ now refer to the Brownian approximation. As with other heavy traffic approximations, the BCP may be accurate even when these conditions 
are not met.

\subsubsection{The Workload Formulation}

To achieve a state space collapse, we reformulate as in Wein. Let $\hat{B}(t)=\sum m_{k} \hat{X}_{k}(t)$, a Brownian motion with drift $\hat{\mu}=\sqrt{n}(1-\rho)$ and variance $\sigma^{2}=\sum m_{k} \gamma_{k}\left(v_{s k}^{2}+v_{d k}^{2}\right)$. The workload formulation (WF) is to find processes $\hat{Z}_{k}, \hat{I}$, and $\hat{L}_{k}$ that are nonanticipating with respect to $\hat{B}$ to minimize (48) subject to

$$
\begin{gathered}
\sum m_{k} \hat{Z}_{k}(t)=\hat{B}(t)-\hat{I}(t)+\hat{\mathcal{L}}(t), \\
\hat{\mathcal{L}}(t)=\sum m_{k} \hat{L}_{k}(t) \\
\hat{Z}_{k}(t) \geq 0, \text { and } \\
\hat{I} \text { and } \hat{L}_{k} \text { nondecreasing. }
\end{gathered}
$$

As the following theorem asserts, WF is a relaxation of BCP with the same optimal objective function value, and we can solve WF instead of BCP.

Theorem 1. (i) Every feasible policy $\hat{Y}$ for BCP corresponds to a feasible policy $(\hat{Z}, \hat{I}, \hat{L})$ for $W F$ of equal cost. (ii) Every optimal policy $(\hat{Z}, \hat{I}, \hat{L})$ for $W F$ corresponds to a feasible policy $\hat{Y}$ for BCP of equal cost.

A proof is given in Section 3.3.4 after the optimal policy is derived.

\subsubsection{Solving the Workload Formulation}

The workload formulation will be solved in two steps. First, an optimal $\hat{Z}$ and $\hat{L}$ is found in terms of $\hat{I}$, then an optimal $\hat{I}$ is found. Define $\hat{W}(t)=\sum m_{k} \hat{Z}_{k}(t)$ and classes $i$ and $j$ satisfying $\hat{h} \equiv \hat{h}_{i} \mu_{i}=\min \left\{\hat{h}_{k} \mu_{k}\right\}$ and $\hat{l} \equiv \hat{l}_{j} \mu_{j}=\min \left\{\hat{l}_{k} \mu_{k}\right\}$. It is optimal to 
set $\hat{\mathcal{L}}(t)=-\inf _{0 \leq s \leq t}\{\hat{B}(t)-\hat{I}(t)\}$, since this is the minimal $\hat{\mathcal{L}}$ that satisfies $\hat{W}(t) \geq 0$, implied by (55), and cost is increasing in $\hat{\mathcal{L}}$. Then the optimal $\hat{Z}$ at each $t$ is a solution to the linear program

$$
\begin{aligned}
\min & \sum \hat{h}_{k} \hat{Z}_{k}(t) \\
\text { subject to } & \sum m_{k} \hat{Z}_{k}(t)=\hat{B}(t)-\hat{I}(t)+\hat{\mathcal{L}}(t) \text { and } \\
& \hat{Z}_{k}(t) \geq 0,
\end{aligned}
$$

namely, the $h \mu$ rule

$$
\hat{Z}_{k}^{*}(t)= \begin{cases}\mu_{k} \hat{W}(t), & k=i \\ 0, & k \neq i\end{cases}
$$

The optimal cost is $\hat{h} \hat{W}(t)$.

Similarly, the optimal $\hat{L}$ at each $t$ is a solution to

$$
\begin{aligned}
\min & \sum \hat{l}_{k} \hat{L}_{k}(t) \\
\text { subject to } & \sum m_{k} \hat{L}_{k}(t)=\hat{\mathcal{L}}(t) \text { and } \\
& \hat{L}_{k}(t) \geq 0,
\end{aligned}
$$

namely, the " $l \mu$ " rule

$$
\hat{L}_{k}^{*}(t)= \begin{cases}\mu_{k} \hat{\mathcal{L}}(t), & k=j \\ 0, & k \neq j .\end{cases}
$$

Note that $\hat{L}^{*}$ is nondecreasing as required by (56). The optimal cost is $\hat{l} \hat{\mathcal{L}}(t)$.

Next we solve for $\hat{I}$. Substituting $\hat{Z}^{*}$ and $\hat{L}^{*}$ into WF gives

$$
\min \limsup _{T \rightarrow \infty} \frac{1}{T} E\left[\int_{0}^{T} \hat{h} \hat{W}(t) \mathrm{d} t+\hat{l} \hat{\mathcal{L}}(T)\right]
$$

$$
\text { subject to } \hat{W}(t)=\hat{B}(t)-\hat{I}(t)+\hat{\mathcal{L}}(t) \text { and }
$$




$$
\hat{\mathcal{L}}(t)=-\inf _{0 \leq s \leq t}\{\hat{B}(t)-\hat{I}(t)\} .
$$

A natural choice for $\hat{I}$ is to keep $\hat{W}$ in the interval [0, $\hat{c}]$; standard arguments (see, for example, Menaldi and Robin 1984 and Taksar 1985) can be used to show that such a policy is optimal. Let $\hat{I}$ be the unique function satisfying $\hat{I}(t)=\sup _{0 \leq s \leq t}[\hat{B}(s)+$ $\hat{\mathcal{L}}(s)-\hat{c}]^{+},(67)$, and $\hat{I}$ increasing only when $\hat{W}(t)=\hat{c}$. Then $\hat{W}$ is a regulated Brownian motion (RBM) on $[0, \hat{c}]$ with the same parameters as $\hat{B}$. Let us begin with the case $\hat{\mu}>0$, corresponding to $\rho<1$. It is well known (see Harrison 1985) that the steady-state p.d.f. of $\hat{W}$ is $p(x)=\hat{\nu} e^{\hat{\nu} x} /\left(e^{\hat{\nu} \hat{c}}-1\right), 0 \leq x \leq \hat{c}$, where $\hat{\nu}=2|\hat{\mu}| / \sigma^{2}$, and the lower control rate is

$$
\beta \equiv \lim _{t \rightarrow \infty} \frac{\hat{\mathcal{L}}(t)}{t}=\frac{\hat{\mu}}{e^{\hat{\nu} \hat{c}}-1} .
$$

If we restrict ourselves to RBM policies, then $(65)$ - (67) reduces to finding $\hat{c}$ to minimize

$$
\begin{aligned}
\phi(\hat{c}) & =\int_{0}^{\hat{c}} \hat{h} x p(x) \mathrm{d} x+\hat{l} \beta \\
& =\int_{0}^{\hat{c}} \frac{\hat{h} \hat{\nu} x e^{\hat{\nu} x}}{e^{\hat{\nu} \hat{c}}-1} \mathrm{~d} x+\frac{\hat{l} \hat{\mu}}{e^{\hat{\nu} \hat{c}}-1} \\
& =\frac{\hat{l} \hat{\mu}+\hat{h} \hat{c} e^{\hat{\nu} \hat{c}}}{e^{\hat{\hat{c}} \hat{c}}-1}-\frac{\hat{h}}{\hat{\nu}}
\end{aligned}
$$

Setting $\phi^{\prime}(\hat{c})=0$ yields

$$
e^{\hat{\nu} \hat{c}}-\hat{\nu} \hat{c}-1-\hat{\nu} \hat{\mu} \hat{l} / \hat{h}=0
$$

We now reverse the scalings of the parameters in this equation so that it is expressed solely in terms of the original problem parameters. The solution $\hat{c}$ to (70) is an upper control limit for the scaled workload. If we denote the workload in the original system by $W(t)$, where $\hat{W}(t)=W(n t) / \sqrt{n}$, then our proposed idleness policy for the original system is to idle when $W(t)=c=\sqrt{n} \hat{c}$. In terms of $c,(70)$ can be 
written, using $(46)$ and $\hat{\mu}=\sqrt{n}(1-\rho)$, as

$$
e^{\frac{2(1-\rho) c}{\sigma^{2}}}-\frac{2(1-\rho) c}{\sigma^{2}}-1-\frac{2(1-\rho)^{2} l}{\sigma^{2} h}=0
$$

where $h \equiv \min \left\{h_{k} \mu_{k}\right\}$ and $l \equiv \min \left\{l_{k} \mu_{k}\right\}$. This equation can be solved numerically for the proposed workload threshold $c$ in terms of the original problem parameters.

Now consider the case $\hat{\mu}<0$, corresponding to $\rho>1$. The p.d.f. of $\hat{W}$ is $p(x)=\hat{\nu} e^{-\hat{\nu} x} /\left(1-e^{-\hat{\nu} \hat{c}}\right), 0 \leq x \leq \hat{c}$, and the lower control rate is

$$
\beta=\frac{|\hat{\mu}|}{1-e^{-\hat{\nu} \hat{c}}}
$$

The cost rate is

$$
\begin{aligned}
\phi(\hat{c}) & =\int_{0}^{\hat{c}} \frac{\hat{h} \hat{\nu} x e^{-\hat{\nu} x}}{1-e^{-\hat{\nu} \hat{c}}} \mathrm{~d} x+\frac{\hat{l}|\hat{\mu}|}{1-e^{-\hat{\nu} \hat{c}}} \\
& =\frac{\hat{l}|\hat{\mu}|-\hat{h} \hat{c} e^{-\hat{\nu} \hat{c}}}{1-e^{-\hat{\nu} \hat{c}}}+\frac{\hat{h}}{\hat{\nu}}
\end{aligned}
$$

and is minimized at

$$
\begin{gathered}
e^{-\hat{\nu} \hat{c}}+\hat{\nu} \hat{c}-1-\hat{\nu}|\hat{\mu}| \hat{l} / \hat{h}=0, \text { or } \\
e^{\frac{2(1-\rho) c}{\sigma^{2}}}+\frac{2(1-\rho) c}{\sigma^{2}}-1-\frac{2(1-\rho)^{2} l}{\sigma^{2} h}=0 .
\end{gathered}
$$

Finally, for the case $\mu=0$, corresponding to $\rho=1$, the p.d.f. of $\hat{W}$ is $p(x)=1 / \hat{c}$, $0 \leq x \leq \hat{c}$, and the lower control rate is $\beta=\sigma^{2} /(2 \hat{c})$. The cost rate is $\phi(\hat{c})=$ $\hat{h} \hat{c} / 2+\hat{l} \sigma^{2} /(2 \hat{c})$, and is minimized at $\hat{c}=\sqrt{\sigma^{2} \hat{l} / \hat{h}}$, or

$$
c=\sqrt{\sigma^{2} l / h}
$$

These three results are consistent. As $\rho \rightarrow 1, \nu \rightarrow 0$ and the small-exponent approximation $e^{x} \approx 1+x+x^{2} / 2$ can be used in (71) and (75), giving (76). 
We end this section by proving the theorem.

Proof of theorem.

(i) Given $\hat{Y}$ feasible for BCP, let $\hat{L}_{k}$ satisfy $(51), \hat{\mathcal{L}}$ satisfy (54), and $\hat{Z}_{k}$ satisfy (49). Then

$$
\begin{aligned}
\sum m_{k} \hat{Z}_{k}(t) & =\sum m_{k} \hat{X}(t)-\sum \hat{Y}_{k}(t)+\sum m_{k} \hat{L}_{k}(t) \\
& =\hat{B}(t)-\hat{I}(t)+\hat{\mathcal{L}}(t),
\end{aligned}
$$

i.e., (53) holds. Also, (49), (51), and (52) imply (55) - (56), and $(\hat{Z}, \hat{I}, \hat{L})$ is feasible for WF.

(ii) Given $(\hat{Z}, \hat{I}, \hat{L})$ optimal for WF, let $\hat{Y}$ satisfy $(49)$, namely $\hat{Y}_{k}(t)=m_{k}\left[\hat{X}_{k}(t)-\right.$ $\left.\hat{Z}_{k}(t)+\hat{L}_{k}(t)\right]$. Then

$$
\sum \hat{Y}_{k}(t)=\hat{B}_{k}(t)-\sum m_{k} \hat{Z}_{k}(t)+\hat{\mathcal{L}}(t)=\hat{I}(t)
$$

i.e., (50) holds. Substituting $\hat{Y}_{k}$ into the r.h.s. of (51) gives

$$
-\inf _{0 \leq s \leq t}\left\{\hat{Z}_{k}(s)-\hat{L}_{k}(s)\right\}
$$

For $k \neq j, \hat{L}_{k}(s)=0,(79)$ reduces to $\hat{Z}_{k}(0)=0$, and (51) holds. Now consider $k=j$. If $j \neq i$, then $\hat{Z}_{j}(s)=0$ and, since $\hat{L}_{j}$ is nondecreasing, (79) is just $\hat{L}_{j}(t)$, i.e., (51) holds. If $j=i$, then by WF optimality, $\hat{L}_{j}$ increases only at times $s$ when $\hat{\mathcal{L}}(s)$ is increasing. But $\hat{\mathcal{L}}$ is the lower regulator for $\hat{W}$, so at these times $\hat{W}(s)=\hat{Z}_{j}(s)=0$. Since $\hat{Z}_{j}(s) \geq 0$ and $\hat{L}_{j}$ is nondecreasing, it follows that (79) is the largest value of $\hat{L}_{j}$, namely, $\hat{L}_{j}(t)$, and (51) holds. Optimality also ensures that $\hat{I}(0)=0$ and (52) holds, so $\hat{Y}$ is feasible for BCP. 
Table 2: Throughput Iteration for Lost Sales Case 2.

\begin{tabular}{clcc} 
Iteration & Initial $\gamma_{2}$ & $\mathrm{c}$ & Final $\gamma_{2}$ \\
\hline 1 & .45 & 10.8 & .4069 \\
2 & .4069 & 10.5 & .4084 \\
3 & .4084 & 10.5 & .4083
\end{tabular}

\subsubsection{Updating the Throughput}

The Brownian motion variance $\sigma^{2}$ appearing in (70) and (74) depends on the unknown throughputs $\gamma_{k}$. As in Dai and Harrison, we overcome this difficulty by iteratively computing $c$ and $\gamma$. A reasonable initial value is $\gamma_{k}=\rho_{k}$. Given $\gamma_{k}$, compute $\sigma^{2}$, use (71), (75) or (76) to compute $c$, and (68) or (72) to compute $\beta$. To update $\gamma$, recall that all lost sales are attributed to class $j$ by the $l \mu$ rule, so that $\hat{\mathcal{L}}(t)=m_{j} \hat{L}_{j}(t)$ and the lost sales rate for class $j$ is $\lambda_{j} P_{j}=\beta / m_{j}$. From (47), we obtain

$$
\gamma_{j}=\rho_{j}-\beta
$$

It is possible for (80) to give $\gamma_{j}<0$, meaning that there are more lost sales than class $j$ arrivals. A reasonable allocation of these lost sales is to set $\gamma_{j}=0, \beta=\beta-\rho_{j}$, and repeatedly apply (80) to the class with next smallest $l_{k} \mu_{k}$. Using the new $\gamma$, the calculations can be repeated. Convergence is reached rapidly, as demonstrated in Table 2.

\section{Numerical Results}

Dynamic programming value iteration was used to compute optimal policies for undiscounted problems with two and three products. The recurrent states are those below the hedging point, $x \leq x^{*}$. For the lost sales problem, the recurrent class is finite, 
$0 \leq x \leq x^{*}$. For the backorder problem, the state space was truncated. Larger and larger state spaces were tested until the results were insensitive to increasing the state space. State spaces up to about 30 by 30 and up to 2000 value iterations were required to achieve three digit accuracy. The lost sales problem generally ran much faster.

All compatible combinations of switching curves and hedging points listed in Table 1 were tested. These candidate policies were evaluated using a value-iteration scheme to avoid directly solving a large linear system. The LQ and offset LQ switching curves described in Section 1 were also tested, as were the hedging point approximations generated by pure STLA and restless bandit index policies. Finally, for STLA, restless bandit, and LQ switching curves, a one-dimensional search along the switching curve was conducted to find the best hedging point for that switching curve. This data point is used to determine how much of the suboptimality of a policy is due to the switching curve and how much is due to the hedging point. These three switching curves are combined with hedging points by converting the hedging point to a workload threshold (see Section 3.3), then finding the point on the switching curve that matches or exceeds this workload. The $\mu \Delta V$ and offset LQ switching curves require a $K$ dimensional hedging point, not just a a one-dimensional workload, to be specified. Best hedging points were not found for these switching curves.

\subsection{Lost Sales}

Most of the testing was devoted to the two-product lost sales problem. Five test cases are defined in Table 3 . We begin by comparing hedging point approximations. Table 4 shows the hedging points for the test problems. Since $\mu_{k}=1$ in these problems, the workload is just the sum of the hedging point coordinates, $w=\sum x_{k}^{*}$. The suboptimality, measured in terms of the gain per unit time $g / \Lambda$, is also shown. For convenience, all of the hedging points are combined with the STLA switching curve, 
Table 3: Lost Sales Cases $(\mu=1)$.

\begin{tabular}{cccccccccc} 
Case & $\lambda_{1}$ & $\lambda_{2}$ & $\lambda_{3}$ & $h_{1}$ & $h_{2}$ & $h_{3}$ & $s_{1}$ & $s_{2}$ & $s_{3}$ \\
\hline 1 & .4 & .5 & & 1 & 1 & & 60 & 80 & \\
2 & .45 & .45 & & 4 & 1 & & 45 & 45 & \\
3 & .45 & .45 & & 1 & 1 & & 90 & 360 & \\
4 & .3 & .6 & & 1 & 1 & & 120 & 300 & \\
5 & .3 & .4 & & 2 & 1 & & 60 & 40 & \\
\hline 6 & .2 & .3 & .4 & 1 & 1.25 & 1.5 & 40 & 75 & 120
\end{tabular}

Table 4: Lost Sales Hedging Point Approximations.

\begin{tabular}{|c|c|c|c|c|c|c|c|c|c|c|c|}
\hline \multirow[b]{3}{*}{ Case } & \multirow{3}{*}{$\begin{array}{c}\text { Opt. } \\
x^{*}\end{array}$} & \multicolumn{4}{|c|}{ Pure Index } & \multirow{2}{*}{\multicolumn{2}{|c|}{ Allocated }} & \multirow{2}{*}{\multicolumn{2}{|c|}{ Aggregate }} & \multirow{2}{*}{\multicolumn{2}{|c|}{ Brownian }} \\
\hline & & \multicolumn{2}{|c|}{ Restless } & \multicolumn{2}{|c|}{ STLA } & & & & & & \\
\hline & & $x^{*}$ & sub & $x^{*}$ & sub & $x^{*}$ & sub & $w$ & sub & $w$ & sub \\
\hline 1 & $(6,7)$ & $(4,5)$ & $15 \%$ & $(4,4)$ & 21 & $(8,10)$ & $9 \%$ & 9 & $12 \%$ & 12.4 & $0 \%$ \\
\hline 2 & (c. & $(2,4$ & $7 \%$ & $(2$ & 14 & $(4,7$ & $2 \%$ & 5 & 14 & 5 & 70 \\
\hline 3 & ( & $(5,6$ & 41 & $(4$, & 54 & (10 & $23 \%$ & 1 & $5 \%$ & 3.9 & $\%$ \\
\hline 4 & ( & $(4,8$ & 48 & $(4,6$ & 54 & (11 & $15 \%$ & 15 & 99 & 17.9 & $1 \%$ \\
\hline 5 & & $(3$ & $2 \%$ & $(3,3)$ & $5 \%$ & & $8 \%$ & 6 & $5 \%$ & 6.5 & $0 \%$ \\
\hline 6 & & 34 & $29 \%$ & $\overline{3,4)}$ & $8 \%$ & $8,10)$ & $27 \%$ & 9 & $3 \%$ & 3.9 & $2 \%$ \\
\hline
\end{tabular}

except for the pure restless bandit index hedging point, which is combined with its own switching curve. However, the ranking of the suboptimalities was the same for most of the cases when other switching curves were used. Note that the allocated server hedging points shown must be shifted onto the STLA switching curves, maintaining the same workload, before evaluating the policy.

The results show the Brownian hedging point to be the clear winner, both in terms of accuracy of the workload threshold and the resulting gain. If the workload must be broken down into stock levels, the allocated server hedging point is the best candidate. As expected, the allocated server hedging point is too large and the aggregate product too small. Since the pure index policies disregard congestion 
Table 5: Lost Sales Switching Curves.

\begin{tabular}{ccccccccccc} 
& \multicolumn{2}{c}{ LQ } & \multicolumn{2}{c}{ Offset LQ } & \multicolumn{2}{c}{ Restless } & \multicolumn{2}{c}{ STLA } & \multicolumn{2}{c}{$\mu \Delta V$} \\
Case & $w$ & sub & $x^{*}$ & sub & $x^{*}$ & sub & $x^{*}$ & sub & $x^{*}$ & sub \\
\hline 1 & 13 & $1 \%$ & $(8,10)$ & $11 \%$ & $(6,7)$ & $1 \%$ & $(6,7)$ & $0 \%$ & $(8,10)$ & $10 \%$ \\
& 13 & $1 \%$ & & & $(6,7)$ & $1 \%$ & $(6,7)$ & $0 \%$ & & \\
\hline 2 & 11 & $20 \%$ & $(4,7)$ & $13 \%$ & $(5,6)$ & $13 \%$ & $(3,8)$ & $2 \%$ & $(4,7)$ & $6 \%$ \\
& 8 & $9 \%$ & & & $(3,5)$ & $1 \%$ & $(3,6)$ & $0 \%$ & & \\
\hline 3 & 14 & $38 \%$ & $(10,18)$ & $35 \%$ & $(7,7)$ & $15 \%$ & $(6,8)$ & $8 \%$ & $(10,18)$ & $34 \%$ \\
& 20 & $17 \%$ & & & $(9,9)$ & $6 \%$ & $(8,10)$ & $2 \%$ & & \\
\hline 4 & 18 & $9 \%$ & $(11,17)$ & $20 \%$ & $(6,12)$ & $8 \%$ & $(7,11)$ & $1 \%$ & $(11,17)$ & $19 \%$ \\
& 21 & $6 \%$ & & & $(7,14)$ & $6 \%$ & $(8,12)$ & $1 \%$ & & \\
\hline 5 & 7 & $5 \%$ & $(5,5)$ & $16 \%$ & $(3,4)$ & $2 \%$ & $(3,4)$ & $0 \%$ & $(5,5)$ & $15 \%$ \\
& 8 & $4 \%$ & & & $(3,5)$ & $1 \%$ & $(3,5)$ & $0 \%$ & & \\
\hline 6 & 14 & $7 \%$ & $(7,8,10)$ & $26 \%$ & $(4,4,6)$ & $6 \%$ & $(5,4,5)$ & $2 \%$ & $(7,8,10)$ & $27 \%$ \\
& 17 & $2 \%$ & & & $(4,5,6)$ & $4 \%$ & $(6,4,5)$ & $2 \%$ & &
\end{tabular}

from other classes, their hedging points are too small, as predicted in Section 2.3. However, they perform much better in case 5 , where $\rho=0.7$, than the other cases, where $\rho=0.9$.

Next, using the best available hedging point (Brownian if only the workload is needed, allocated server if the stock levels are needed), the switching curves are compared in Table 5. The second row of figures within a case gives the best hedging point for the switching curve, where available. The best hedging point illustrates how much of the suboptimality is due to the switching curve. The results suggest that the best policy is the STLA index combined with the Brownian hedging point. Its gain is within $8 \%$ of optimal for all five test cases. The restless bandit index combined with the Brownian hedging point also does well. Much of the suboptimality for these policies is due to the hedging point, even though we have used the most accurate of the approximations. It appears that finding a good hedging point is more difficult than finding a good switching curve. The potential savings from using index policies is best measured by comparing STLA to the LQ policy. Average suboptimality is 
reduced from up $15 \%$ to $2 \%$.

The offset LQ and $\mu \Delta V$ switching curves perform poorly, possibly because they must use the less accurate server allocation hedging point. The simple LQ switching curve performs slightly better, but has trouble with asymmetric products, such as case 3. Its suboptimality is due to both the switching curve and the Brownian hedging point that is used with it.

The shapes of various switching curves for case 4 are shown in Fig. 1. The STLA curve is slightly closer to the line of symmetry $x_{1}=x_{2}$, i.e., the LQ switching curve, than optimal. The restless bandit curve starts much farther from symmetry and initially is nearly horizontal, reflecting the dominance of the lost sales term in (28). The $\mu \Delta V$ curve is parallel to the symmetry line but shifted too far away. The optimal idleness region is also shown. The curvature of its boundary determines how far the optimal hedging point, $(7,13)$, is from the the pure restless bandit index hedging point, $(4,8)$, the latter lying on the asymptotes of the idleness region.

The optimal switching curve was found to be close to the symmetry line for fairly different products. The curve is particularly insensitive to $s$, with differences of a factor of two scarcely affecting the curve. Significantly different product parameters were found to have the following effects on the switching curve:

1. $s_{1} \ll s_{2}$. The curve is offset toward class $2\left(x_{1}<x_{2}\right)$.

2. $h_{1} \gg h_{2}$. The curve starts at the origin and moves toward $x_{1}<x_{2}$ (slope steeper than one).

3. $\lambda_{1}<\lambda_{2}$. The curve starts at $x_{1}>x_{2}$ (below the symmetry line) and moves to $x_{1}<x_{2}$ (slope steeper than one). If lost sales costs are equal, $s_{1} / \lambda_{1}=s_{2} / \lambda_{2}$, then the curve starts above the symmetry line.

4. Expensive product: $s_{1} \ll s_{2}$ and $h_{1} / s_{1}=h_{2} / s_{2}$. The curve starts at $x_{1}<x_{2}$ 


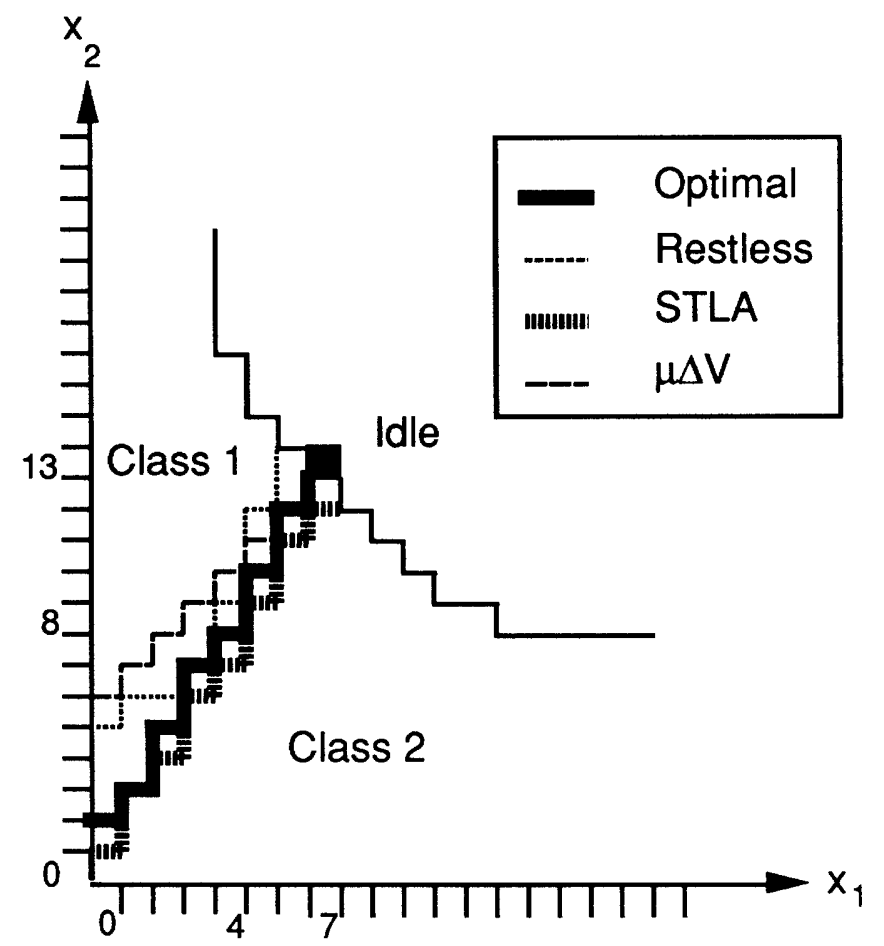

Figure 1: Shape of the Switching Curves- Lost Sales Case 4.

(above the symmetry line) and is nearly horizontal.

We also made a few runs with three products. Case 6 in Tables 4 and 5 is consistent with the results for two products. Recall that the restless bandit index is predicted to become more accurate for a large number of products. The three-product cases we have tested neither support nor refute this prediction.

\subsection{Backorders}

Three backorder cases are described in Table 6. The hedging point approximations and their suboptimality are shown in Table 7. Again, STLA switching curves are combined with the hedging points. The results suggest that the Brownian and LQ hedging points are the most accurate, with Brownian a little better on case 1, which, surprisingly, is the case with the lowest traffic intensity. The aggregate product 
Table 6: Two-Product Backorder Cases $(\mu=1)$.

\begin{tabular}{ccccccc} 
Case & $\lambda_{1}$ & $\lambda_{2}$ & $h_{1}$ & $h_{2}$ & $b_{1}$ & $b_{2}$ \\
\hline 1 & .3 & .4 & 2 & 1 & 10 & 5 \\
2 & .45 & .45 & 1 & 1 & 2 & 4 \\
3 & .45 & .45 & 1.25 & 1 & 4 & 2
\end{tabular}

Table 7: Backorder Hedging Point Approximations.

\begin{tabular}{cccccccccccc} 
& Opt. & Pure STLA & \multicolumn{2}{c}{ Allocated } & \multicolumn{2}{c}{ LQ } & \multicolumn{2}{c}{ Aggregate } & \multicolumn{2}{c}{ Brownian } \\
Case & $x^{*}$ & $x^{*}$ & sub & $x^{*}$ & sub & $x^{*}$ & sub & $w$ & sub & $w$ & sub \\
\hline 1 & $(1,3)$ & $(1,1)$ & $23 \%$ & $(5,5)$ & $46 \%$ & $(1,2)$ & $6 \%$ & 5 & $0 \%$ & 4.2 & $0 \%$ \\
2 & $(4,4)$ & $(0,1)$ & $55 \%$ & $(10,15)$ & $99 \%$ & $(4,6)$ & $5 \%$ & 13 & $15 \%$ & 9.9 & $5 \%$ \\
3 & $(3,5)$ & $(1,0)$ & $48 \%$ & $(13,10)$ & $78 \%$ & $(6,4)$ & $7 \%$ & 12 & $13 \%$ & 9.9 & $7 \%$
\end{tabular}

hedging point also performs fairly well. As expected, the allocated server hedging point is too large and the aggregate product too small.

To compare switching curves, the Brownian approximation is used when only a workload is needed; the LQ hedging point is used when stock levels are needed. Table 8 shows the results, where the second row within each case again gives the best hedging point for the switching curve. The STLA index combined with the Brownian hedging point appears to be the best candidate policy, with suboptimality of $7 \%$ or less. The LQ switching curve (which is symmetrical) combined with the Brownian hedging point also performs well, but may do poorly on more asymmetric products. Case 3 suggests that the LQ hedging point of $(6,4)$ gives a fairly accurate workload (10 versus an optimal workload of 8 ), used with the STLA switching curve in Table 7, but poor stock levels, used with the offset LQ switching curve in Table 8. Although the differences found between switching curves are not very dramatic, the results clearly show that choosing a good hedging point is very important.

Switching curves for case 3 are drawn in Fig. 2. The $\mu \Delta V$ curve is inaccurate 
Table 8: Backorder Switching Curves.

\begin{tabular}{ccccccccc} 
& \multicolumn{2}{c}{ LQ } & \multicolumn{2}{c}{ Offset LQ } & \multicolumn{2}{c}{ STLA } & \multicolumn{2}{c}{$\mu \Delta V$} \\
Case & $w$ & sub & $x^{*}$ & sub & $x^{*}$ & sub & $x^{*}$ & sub \\
\hline 1 & 5 & $7 \%$ & $(1,2)$ & $8 \%$ & $(1,4)$ & $0 \%$ & $(2,1)$ & $11 \%$ \\
& 4 & $3 \%$ & & & $(1,3)$ & $0 \%$ & & \\
\hline 2 & 10 & $6 \%$ & $(4,6)$ & $7 \%$ & $(5,5)$ & $5 \%$ & $(3,7)$ & $12 \%$ \\
& 9 & $5 \%$ & & & $(4,5)$ & $4 \%$ & & \\
\hline 3 & 10 & $7 \%$ & $(6,4)$ & $10 \%$ & $(2,8)$ & $7 \%$ & $(7,3)$ & $15 \%$ \\
& 8 & $5 \%$ & & & $(2,6)$ & $5 \%$ & &
\end{tabular}

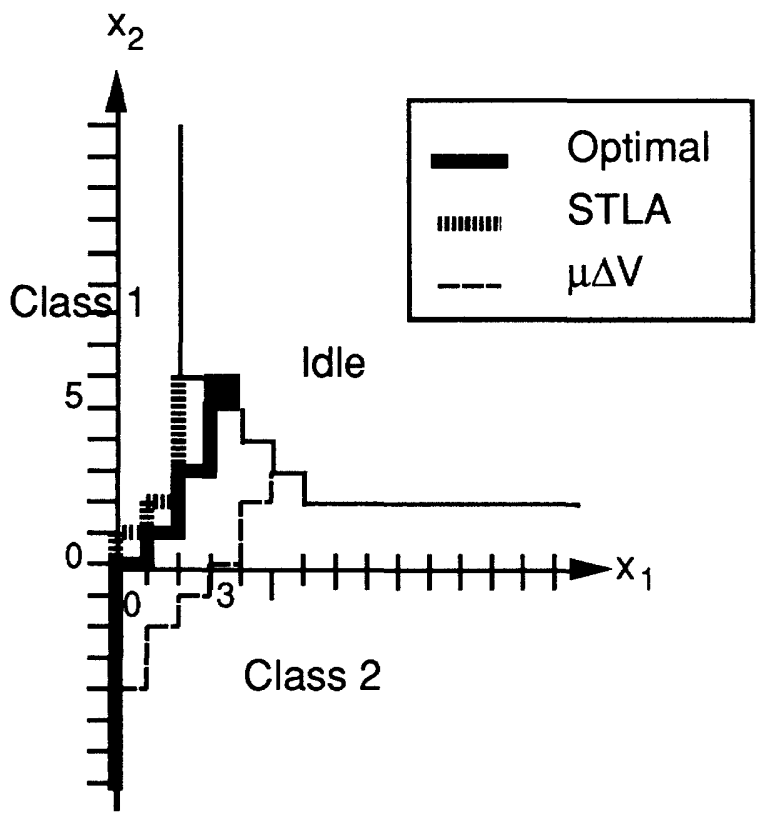

Figure 2: Shape of the Switching Curves- Backorder Case 3. 
primarily because of the LQ hedging point, $(6,4)$, on which it is based. The STLA curve is slightly more asymmetrical than the optimal curve. In general, backorder curves are much more sensitive to asymmetric products, specifically to differences in $\lambda$ and $h$. The $25 \%$ difference in holding costs in case 3 is responsible for moving the hedging point from symmetry to $(3,5)$. Larger differences in $h$, such as in case 1 , result in a hedging point near the axis. This explains the good performance of the "modified $h \mu$ " rule reported in Wein, where hedging points one unit away from the axis, e.g., $\left(x_{1}, 1\right)$, were used.

\section{Concluding Remarks}

We have considered a scheduling problem for a multiclass make-to-stock queue in both the backorder and lost sales cases. Since the optimal solution can only be numerically computed when there are a small number of products, our goal was to derive a scheduling policy, which consists of a hedging point that dictates when to idle and an index policy that specifies which product to serve, that performs well and can be easily computed when there are a large number of products.

Our numerical results suggest that the STLA index policy derived by Zipkin (1990) coupled with the hedging point derived by a Brownian analysis performs very well, at least for two- and three-product problems. Of all the index policies and hedging points considered in this paper, these two are the only ones for which the exponential assumption can be easily relaxed. Our numerical results also suggest that both a good hedging point and a good switching curve are required to attain performance that is close to optimal. Moreover, good hedging points appear to be harder to derive than good switching curves.

We did not attempt any numerical computations for problems with a large number 
of products because the suboptimality of our proposed policies cannot be calculated in these cases. It is possible that the accuracy of the Brownian hedging point would degrade with more products, since it is based on the $h \mu$ rule, which holds all inventory in one class. However, a Brownian workload threshold can be computed using any desired inventory mix, not just the mix dictated by the $h \mu$ rule. For example, Section 9 of Wein obtains a hedging point for the backorder case by performing a Brownian analysis under the LQ policy. This alternative hedging point may be more accurate for large problems that are relatively symmetric.

We noticed a relationship between the Gittens index for the multi-arm bandit problem and the restless bandit index that may be useful for other research. The Gittens index measures the value of playing an arm (serving a class) given that there are other arms of equal value, so that when its value drops we can "retire" to another arm of equal value. For restless problems, the arms change state while passive and there is no constant retirement value. As a result, the Gittens index may not be meaningful. The Gittens index for the discounted, backorder version of our problem, computed in Veatch (1992), is not monotonic and does not produce a coherent policy. Whittle's restless bandit index assumes that the cost of the server is constant over time, and that each product can use the server whenever it is cost-effective. For our problem, these assumptions are only accurate when there are many classes and many servers (or one server and many low utilization classes). Another approach is to compute a Gittens index using a variable retirement cost, $M\left(x_{k}, \nu\right)$, that depends on the inventory $x_{k}$, as well as the "base" retirement cost $\nu$. The traditional Gittens index uses $M\left(x_{k}, \nu\right)=\nu$. Using $M\left(x_{k}, \nu\right)=V\left(x_{k}, \nu\right)$, the optimal value function for the single-product subproblem with server cost $\nu$ (see Section 3), gives the restless bandit index. In other words, the restless bandit index can be defined as a Gittens index with variable retirement cost. The question is how to specify $M\left(x_{k}, \nu\right)$ so that the Gittens index will be nondecreasing and produce accurate policies. Computing such indices numerically should be possible; one approach is found in Taylor (1968). 
The restless bandit and generalized Gittens index may also be useful for attacking the related problem with set-up costs. When set-up costs are added, the state of the dynamic program must be augmented with the class currently being produced. The form of the optimal policy becomes much more complex, involving lot-sizing and scheduling, and good approximations have not been found. An index policy could be constructed by computing two indices for each class, measuring the value of starting and stopping production of that class.

\section{Acknowledgements}

We acknowledge helpful discussions with Avi Mandelbaum and Vien Nguyen. This research is supported by National Science Foundation grant DDM-9057297.

\section{References}

Dai, J.G. and J.M. Harrison 1991. Steady-State Analysis of RBM in a Rectangle: Numerical Methods and a Queueing Application. Annals Appl. Prob. 1, 16-35.

Ha, A.Y. 1992. Optimal Control Policies for Some Production/Inventory Systems. Ph.D. dissertation, Stanford University, Stanford, CA.

Harrison, J.M. 1985. Brownian Motion and Stochastic Flow Systems. John Wiley and Sons, New York.

Harrison, J.M. 1988. Brownian Models of Queueing Networks with Heterogeneous Customer Populations, in W. Fleming and P.L. Lions (eds.), Stochastic Differential Systems, Stochastic Control Theory and Applications, IMA Vol. 10, Springer-Verlag, New York, 147-186. 
Klimov, G.P. 1974. Time Sharing Service Systems I. Th. Appl. Prob. 19, 532-551.

Krichagina, E.V., S.X.C. Lou and M.I. Taksar 1992. Double Band Policy for Stochastic Manufacturing Systems in Heavy Traffic. Working paper, State Univ. of New York, Stony Brook.

Menaldi, J.L. and M. Robin 1984. Some Singular Control Problems with Long Term Average Criterion. Lecture Notes in Control and Information Sciences, Springer-Verlag, New York, 424-432.

Taylor, H.M. 1968. Optimal Stopping of a Markov Process. Annals Math. Stat. 39, 1333-1344.

Taksar, M.I. 1985. Average Optimal Singular Control and a Related Stopping Problem. Math. Oper. Res. 10,63-81.

Veatch, M.H. 1992. Queueing Control Problems for Production/Inventory Systems. Ph.D. dissertation, Sloan School of Management, MIT, Cambridge.

Weber, R.R. and G. Weiss 1990. On an Index Policy for Restless Bandits. J. Appl. Prob. 27, 637-648.

Wein, L.M. 1992. Dynamic Scheduling of a Multiclass Make-to-Stock Queue. Oper. Res. 40, 724-735.

Whittle, P. 1988. Restless Bandits: Activity Allocation in a Changing World. In A Celebration of Applied Probability, ed. J. Gani, J. Appl. Prob. 25A, 287-298.

Zheng, Y. and P. Zipkin 1990. A Queueing Model to Analyze the Value of Centralized Inventory Information. Oper. Res. 38, 296-307.

Zipkin, P. 1990. An Alternative Dynamic Scheduling Policy. Working notes, Graduate School of Business, Columbia Univ., New York.

Zipkin, P. 1992. Performance of the Smallest-Inventory (or Longest-Queue) Policy. Working paper, Graduate School of Business, Columbia Univ., New York. 